\title{
Real-Life Experience of Monoclonal Antibody Treatments in Chronic Rhinosinusitis with Nasal Polyposis
}

\author{
Eva C. Meier ${ }^{a}$ Peter Schmid-Grendelmeier ${ }^{b, c}$ Urs C. Steiner $^{d}$ \\ Michael B. Soyka ${ }^{a}$ \\ ${ }^{a}$ Department of Otorhinolaryngology Head and Neck Surgery, University of Zurich and University Hospital Zurich, \\ Zurich, Switzerland; 'bepartment of Dermatology, Allergy Unit, University of Zurich and University Hospital Zurich, \\ Zurich, Switzerland; ' Christine Kühne Center for Allergy Research and Education CK-CARE, Davos, Switzerland;

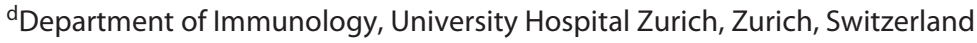

\section{Keywords}

Chronic rhinosinusitis with nasal polyposis · Monoclonal antibody · Benralizumab · Mepolizumab · Omalizumab

\begin{abstract}
Introduction: Few studies assess biologicals such as, omalizumab, mepolizumab, benralizumab, and dupilumab in patients suffering from chronic rhinosinusitis with nasal polyposis (CRSwNP). The reported success rate in these studies differ, and it remains uncertain if there are any biomarkers to predict successful therapy. Our aim was to analyze the therapeutic outcome in a real life setting and to identify predictive biomarkers for successful treatment. Methods: Data from patients with CRSwNP treated with a monoclonal antibody between November 2014 and January 2020 were analyzed retrospectively. Improvement in the polyp score and clinical symptoms like nasal obstruction, sense of smell, nasal discharge, and facial pain were evaluated. Other characteristics, including use of nasal or systemic steroids, comorbidities, previous history of sinus surgery, eosinophilia tissue, blood values (eosinophils, total immunoglobulin E, eosinophilic cationic protein, and interleukin 5), and allergic sensitization in serum were also investigated to identify pos-
\end{abstract}

karger@karger.com www.karger.com/iaa

Karger $\stackrel{\text { ' }}{5}$

GOPEN ACCESS
(C) 2021 The Author(s)

Published by S. Karger AG, Basel

This is an Open Access article licensed under the Creative Commons Attribution-NonCommercial-4.0 International License (CC BY-NC) (http://www.karger.com/Services/OpenAccessLicense), applicable to the online version of the article only. Usage and distribution for commercial purposes requires written permission. sible predictive biomarkers. Results: Forty-eight treatments in 29 patients $(\mathrm{m} / \mathrm{f}=15 / 14)$ aged $27-70$ years were reviewed. Treatments with mepolizumab showed the best success rates $(78.9 \%)$, followed by omalizumab (50\%) and benralizumab treatments (50\%). However, a correlation between biomarkers and treatment success could not be found. Discussion/Conclusion: Treatment of CRSwNP with biologicals is a promising option for severe cases not responding to conventional therapy, including difficult-to-treat patients. Predictive biomarkers for a successful treatment could not be identified, but the reduction of eosinophilic cationic protein was linked with the response.

(C) 2021 The Author(s)

Published by S. Karger AG, Basel

\section{Introduction}

Chronic rhinosinusitis (CRS) is a common health problem with an overall prevalence of $10.9 \%$ in Europe [1]. According to endoscopic findings, CRS can clinically be divided into CRS without nasal polyposis (CRSsNP) and CRS

Edited by: H.-U. Simon.
Michael B. Soyka

Department of Otorhinolaryngology Head and Neck Surgery, University of Zurich and University Hospital Zurich

Frauenklinikstrasse 24, CH-8091 Zurich (Switzerland)

Michael.Soyka@usz.ch 
with nasal polyposis (CRSwNP). Current treatment options for CRS including rinsing, local corticosteroids, systemic corticosteroids, short- and long-term antibiotics, and functional sinus surgery can be insufficient and frustrating [2].

Previous studies have shown that a monoclonal antibody (mAB) like omalizumab, mepolizumab, or dupilumab could be an additional treatment option for CRSwNP in patients with and without comorbid asthma [3-8]. Others were unable to show a benefit in the therapy of CRSwNP with omalizumab or mepolizumab, respectively $[9,10]$. It also remains uncertain, if there are any predictive biomarkers for successful therapy, or how long the $\mathrm{mAB}$ should be given.

The first aim of this study was to analyze the therapeutic outcome of $\mathrm{mAB}$ treatments in a "real-life" setting, including the difficulty to treat CRS patients with a tendency for recurring nasal polyposis even after sinus surgery. Our second aim was to identify possible predictive biomarkers for successful therapy.

\section{Materials and Methods}

This is a retrospective description and analysis of patients with CRSwNP, who received therapy with 1 of the following mABs: omalizumab, mepolizumab, or benralizumab, at the University Hospital Zurich between November 2014 and January 2020. This study was approved by the Ethical Committee of the Canton $\mathrm{Zu}$ rich (KEK-2019-00495). Every patient signed a general informed consent, or in case of additional diagnostics due to the study, a study specific consent was obtained.

Medical files were reviewed regarding: the type of $\mathrm{mAB}$ used, the duration of treatment, the dosage, change in the polyp score, change in clinical symptoms such as nasal obstruction, sense of smell, nasal discharge, and facial pain. Other characteristics, including use of nasal or systemic steroids, comorbidities, as well as, use of pre-therapeutic induction therapy (as described below) were also assessed. Previous history of sinus surgery was analyzed, including histological evidence of eosinophilia, blood values (total immunoglobulin E [IgE] and eosinophils), eosinophilic cationic protein (ECP), interleukin (IL)-5, specific serum IgE to seasonal and perennial allergens, and reported adverse events as shown in the Supporting Information: see online suppl. Table 1; for all online suppl. material, see www.karger.com/doi/10.1159/000514262.

The polyp score was determined by means of endoscopy for each side separately before every period of treatment, and during treatment according to the criteria in the supporting information: online suppl. Table 2 . The changes in clinical symptoms were evaluated based on the medical history and at the most recent consultation then classified into 5 categories as shown in the supporting information: online suppl. Table 3.

Comorbidities along with asthma diagnosis (regardless of disease severity) and aspirin intolerances (if available, by provocation) were recorded separately to identify patients with aspirinexacerbated respiratory disease. The results are available in the supporting information. Moreover, previous history of endoscop-

Monoclonal Antibodies for Chronic

Rhinosinusitis with Nasal Polyposis ic sinus surgery, in addition to every sinus surgery in the period before or during the treatment with a $\mathrm{mAB}$, was considered. Meanwhile, induction therapy was defined as surgery or systemic steroids initiated in the 3 months before $\mathrm{mAB}$ treatment.

According to the WHO definition, eosinophilic counts of more than $0.5 \mathrm{G} / \mathrm{L}$ was defined as peripheral eosinophilia [11]. Reference values for IgE, ECP, and IL-5 in our laboratory are shown in the supporting information: online suppl. Table 4.

Allergic sensitization in serum was recorded in the period before and during $\mathrm{mAB}$ treatment, and then classified into perennial and seasonal allergies [12]. The results are available in the supporting information. Adverse events spontaneously reported by the patient were noted by the medical team; however, a systematic survey on the occurrence of these events was not conducted.

All patients were diagnosed with CRSwNP according to the criteria of the European Position Paper on Rhinosinusitis and Nasal Polyps [2] and had failed standard treatment, and were kept on topical steroids and nasal saline rinses throughout the therapy. $\mathrm{Pa}$ tients treated with a $\mathrm{mAB}$ presented with a difficult-to-treat form of CRSwNP with a tendency for recurring nasal polyposis after sinus surgery. Hence, not only a reduction, but also a stabilization of the total nasal polyp score value, in the context of a good clinical control, was defined as successful treatment with regard to polyp size; that is, a patient who had undergone induction therapy might start with grade 0 or 1 polyps and keep this good score throughout the study and still be regarded as a success since no deterioration was detected. An improvement of 1 or more points in clinical symptoms was also defined as successful treatment.

Each patient, for every treatment received, was classified into 3 categories: successful treatment (reduction or stabilization of polyp size and improvement of subjective complaints), partially successful treatment (either reduction or stabilization of polyp size or improvement of subjective complaints), and unsuccessful treatment (increase of polyp size and no improvement of subjective complaints).

\section{Statistical Analysis}

Statistical analysis was performed using SPSS software version 25.0 (IBM), and a 2 -sided $p$ value of $<0.05$ was considered significant. The normality of distribution was checked using the Kolmogorov-Smirnov test. The Mann-Whitney $U$ test was used to compare non-normally distributed continuous variables, whereas Fisher's exact tests were performed for categorical data. The Wilcoxon signed rank test was used for paired data. ANOVA and Kruskal-Wallis test was used to compare multiple groups.

\section{Results}

\section{Baseline Characteristics}

Twenty-nine patients received a total of 48 treatments. Nine patients received more than 1 treatment, which differed, regarding the type of $\mathrm{mAB}$ used or dosage (details below).

Out of 48 treatment cases in total, 3 were excluded from the analysis - 1 due to sinus surgery during the treatment period and another 2 cases were excluded because 
of omalizumab and mepolizumab treatment, respectively - and were primarily performed for asthma and chronic otitis media, where polyps or CRS-symptoms were absent during the treatment period. Nevertheless, we can say that mepolizumab improved symptoms of asthma and chronic otitis media in this patient. The patients' characteristics are summarized in Table 1.

Each $\mathrm{mAB}$ therapy was given subcutaneously at the dosage stipulated by the Swiss medical board and upon confirmation by the health insurer that they would meet the treatment costs. The mepolizumab dosages were always $100 \mathrm{mg}$ every 4 weeks, while benralizumab and omalizumab dosages differed individually. In the case of benralizumab, 5 cases were treated with a dosage of 30 mg every 4 weeks, and in 1 case $30 \mathrm{mg}$, every 8 weeks. The dosages of omalizumab were adjusted according to IgE levels in serum, patients' weight, and symptom control. Therefore, dosages varied from $150 \mathrm{mg}$ every 4 weeks in 1 patient, to $600 \mathrm{mg}$ every 4 weeks in 3 patients.

\section{Effects of $m A B$ s on Symptoms and Findings}

In total 14 (31.1\%) out of 45 biological treatments were successful. Analyzed individually, the success rates of 6 benralizumab treatments (duration $0-17$ months, median 3.0), 19 mepolizumab treatments (duration 1-25 months, median 7.0), and 20 omalizumab treatments (duration 0-44 months, median 6.5) are shown in Figure 1. However, 4 omalizumab treatments were switched to mepolizumab and vice versa, 1 mepolizumab treatment, and 2 benralizumab treatments were switched to omalizumab (shown in Fig. 2). These therapy changes were made on a case-by-case basis due to insufficient therapeutic success.

In 35 cases the polyp score was determined before and during treatment. It initially ranged from 0 to 8 (median 2.00). The differences between the total polyp scores initially and the total polyp scores after therapy ranged from -5 to 6 (median 0.00 ). It is worth noting that negative counts stand for a reduction in the polyp score and positive counts represent an increase in the polyp score. Twenty-one $(60.0 \%)$ cases showed either an improvement $(57.1 \%)$ or a stabilization $(42.9 \%)$.

In the mepolizumab group, 17 cases initially ranged from 0 to 8 (median 2.00) with a nonsignificant change ranging from -5 to 3 (median 0.00 ) during therapy ( $p=$ 0.58 , Wilcoxon signed rank test). Nine $(52.9 \%)$ cases showed either an improvement $(55.6 \%)$ or a stabilization $(44.4 \%$, each polyposis grade 0,1 , or 2 ) in the polyp score (shown in Fig. 3).

In the omalizumab group (16 cases), the polyp score initially ranged from 0 to 6 (median 1.50) with a nonsig-
Table 1. Patients' characteristics

\section{Patients' characteristics}

Male, $n(\%)$

Female, $n(\%)$

$15(53.6)$

Age (mean), years

$13(46.4)$

Asthma bronchiale, $n(\%)$

NSAID intolerance, $n(\%)$

$27(96.4)$

$17(60.7)$

$25(89.3)$

Surgery due to CRS, $n(\%)$

$9(36.0)$

7 (28.0)

4 (16.0)

$2(8.0)$

$2(8.0)$

$1(4.0)$

6

Evidence of histological eosinophilia if a

mucosal biopsy was performed, $n(\%)$

Perennial sensitization, $n(\%)$

$21(100.0)$

$9(64.3)$

Seasonal sensitization, $n(\%)$

$12(85.7)$

CRS, chronic rhinosinusitis; NSAID, nonsteroidal anti-inflammatory drug.

nificant change ranging from -2 to 6 (median 0.00 ) during therapy ( $p=0.49$, Wilcoxon signed rank test). Nine (56.3\%) cases showed either an improvement $(66.7 \%)$ or stabilization $(33.3 \%$, each polyposis grade 0,1 , or 2$)$ in the total polyp score (shown in Fig. 3). The changes in clinical symptoms like nasal obstruction, smell disturbance, nasal discharge, and facial pain for each $\mathrm{mAB}$ are shown in Figure 4 .

\section{Additional Treatments}

In 22 cases, a systemic steroid was prescribed at least once as a salvage therapy for a few days due to worsening clinical symptoms. The frequency of systemic steroid courses ranged from 1 to 5 (median 1.00) per year in the mepolizumab group, and from 1 to 4 (median 2.00) in the omalizumab group. The rates of treatment successes to quantity of steroid courses, per year are shown in the Supporting Information (online suppl. Tables 5 and 6).

Eighteen (40.0\%) treatments were combined with a pre-therapeutic induction therapy. Sixteen treatments included systemic steroids and 2 included functional sinus surgery. The duration of the induction therapies was chosen individually. Regarding systemic steroids, treatment varied from 3 days to 112 days with dosages ranging from 2.5 to $100 \mathrm{mg}$ daily. Patients started with a dose between 10 and $100 \mathrm{mg}$ that was gradually reduced. For example, an initial daily dose of $20 \mathrm{mg}$ was halved every 7 days. The 
Fig. 1. Treatment success in the benralizumab group, mepolizumab group, or omalizumab group.
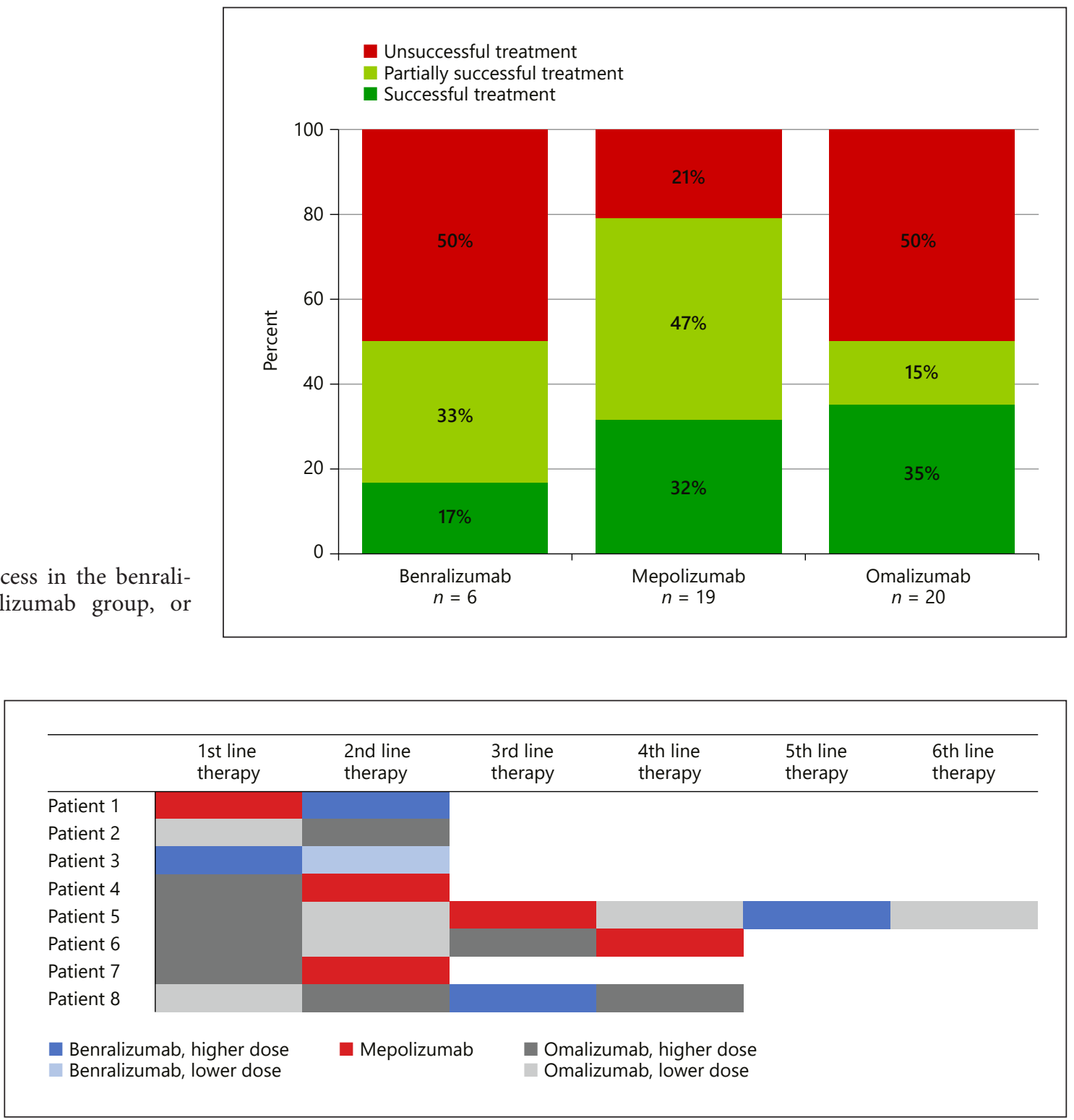

Fig. 2. Sequence of different $\mathrm{mAB}$ therapies in patients who received $>1 \mathrm{mAB}$. Different shades of the same color indicate lower (lighter) or higher (darker) dose of the same $\mathrm{mAB}$.

total polyp score in patients with steroid induced therapy ranged prior to $\mathrm{mAB}$ initiation from 0 to 6 and was 0 in both patients undergoing sinus surgery prior to $\mathrm{mAB}$ therapy. The median change in the total polyp score with steroid-induced treatments and in treatments without induction therapy was 0.00 , respectively.

In the mepolizumab group, the rate of successful or partially successful treatments was $87.5 \%$ in cases with steroid-induced therapy, and $66.7 \%$ in cases without induction therapy. Notwithstanding, the difference of $20.8 \%$ between the two groups, it was not significant $(p=$

Monoclonal Antibodies for Chronic Rhinosinusitis with Nasal Polyposis
0.34 , Fisher's exact test). The two treatments with an induction therapy in the form of functional sinus surgery combined with mepolizumab, were partially successful, as they showed improved clinical symptoms. The rate of successful or partially successful treatments in the omalizumab group was $50.0 \%$ for both steroid-induced cases and cases without induction therapy.

\section{Blood Markers}

Neither maximum values of blood eosinophil counts, nor maximum IgE values, or pre-therapeutic IL-5-values 


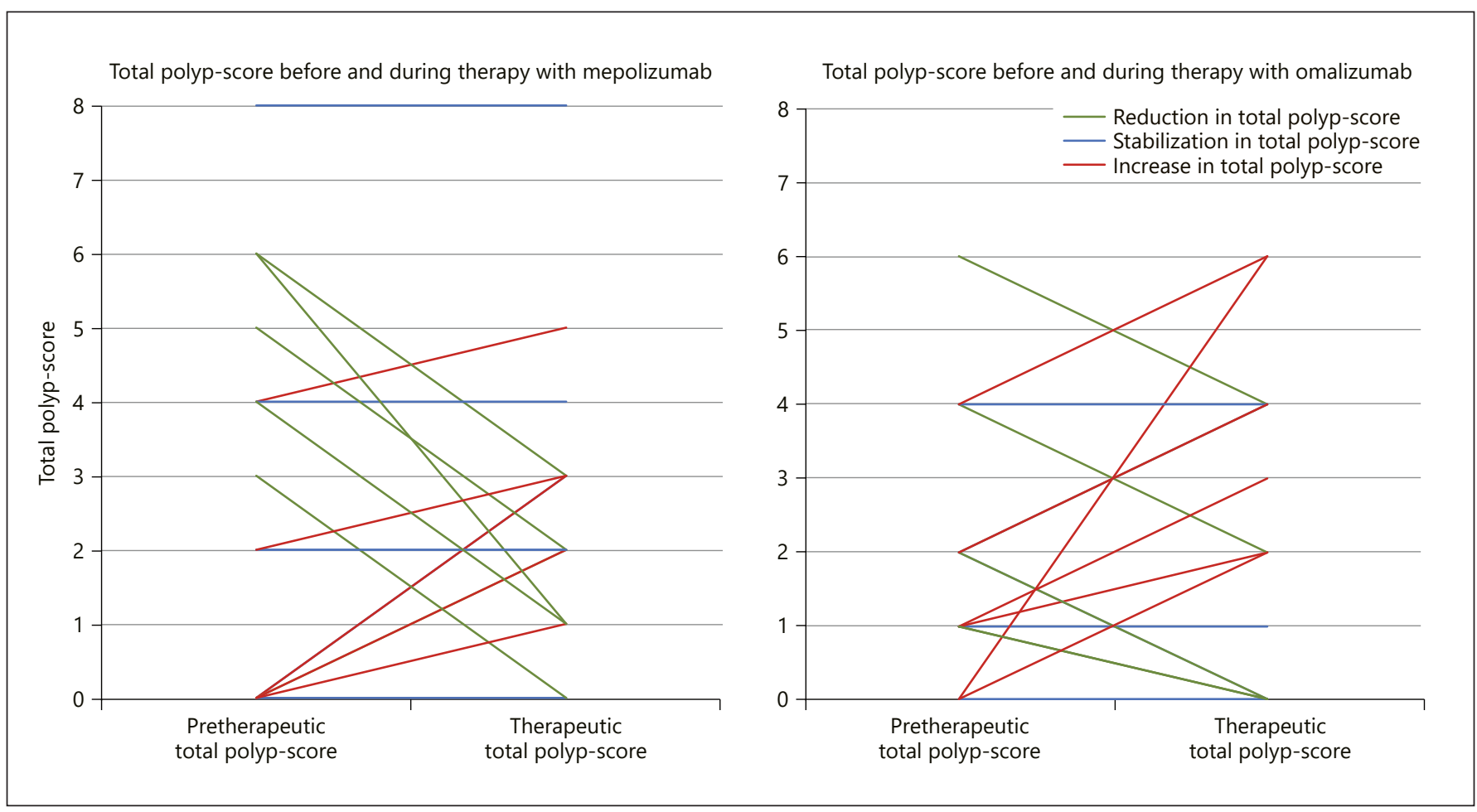

Fig. 3. Change in the total polyp score per patient during therapy with mepolizumab or omalizumab.

could be linked to success rates. However, the history of ECP values was recorded in 13 cases, where a trend toward more successful treatment outcomes could be shown in the mepolizumab treatment group with a decrease in ECP values during therapy ( $p=0.14$, Fisher's exact test).

\section{Adverse Events}

Two patients reported adverse events. One patient from the mepolizumab group reported abdominal pain and fatigue during the treatment. Fatigue also was reported by 1 patient undergoing omalizumab treatment. These side effects led to a therapy-stop in the first patient and to a switch to mepolizumab in the second. No serious adverse events were reported in this study.

\section{Discussion}

The literature and research on $\mathrm{mAB}$ treatments in CRSwNP are scarce. To our knowledge, this is the largest study in a "real-life" setting including difficult-to-treat CRSwNP patients, and also the first one to include 3 different $\mathrm{mABs}$. Our study demonstrated that mepolizumab treatments had the best success rate (78.9\%), followed by omalizumab and benralizumab. However, predictive biomarkers for a successful treatment could not be identified.

Despite limited statistical testing in our study, it seems that the changes in the polyp score during mepolizumab treatment were not as good as in 2 studies from Gevaert et al. [3] and Bachert et al. [6] that reported significant polyp score reduction compared to placebo. Nevertheless, our study still fared better than Chan et al. [10], which could not show a change in the polyp score. It should be noted that the dosage of mepolizumab in the first 2 studies was higher ( $750 \mathrm{mg}$ every 4 weeks), while in the third and our study, a typical clinical dosage of $100 \mathrm{mg}$ every 4 weeks was used. Additionally, in the first 2 studies, the drug was intravenously administered, compared to subcutaneous injections in our and the Chan et al. [10] study, which could potentially explain the different results. Furthermore, unlike other clinical trials, our patients were not strictly screened and considering that we used the Swiss indication guidelines to stay "on-label" as payment was granted by the insurance, we could not increase doses or change the route of administration. 


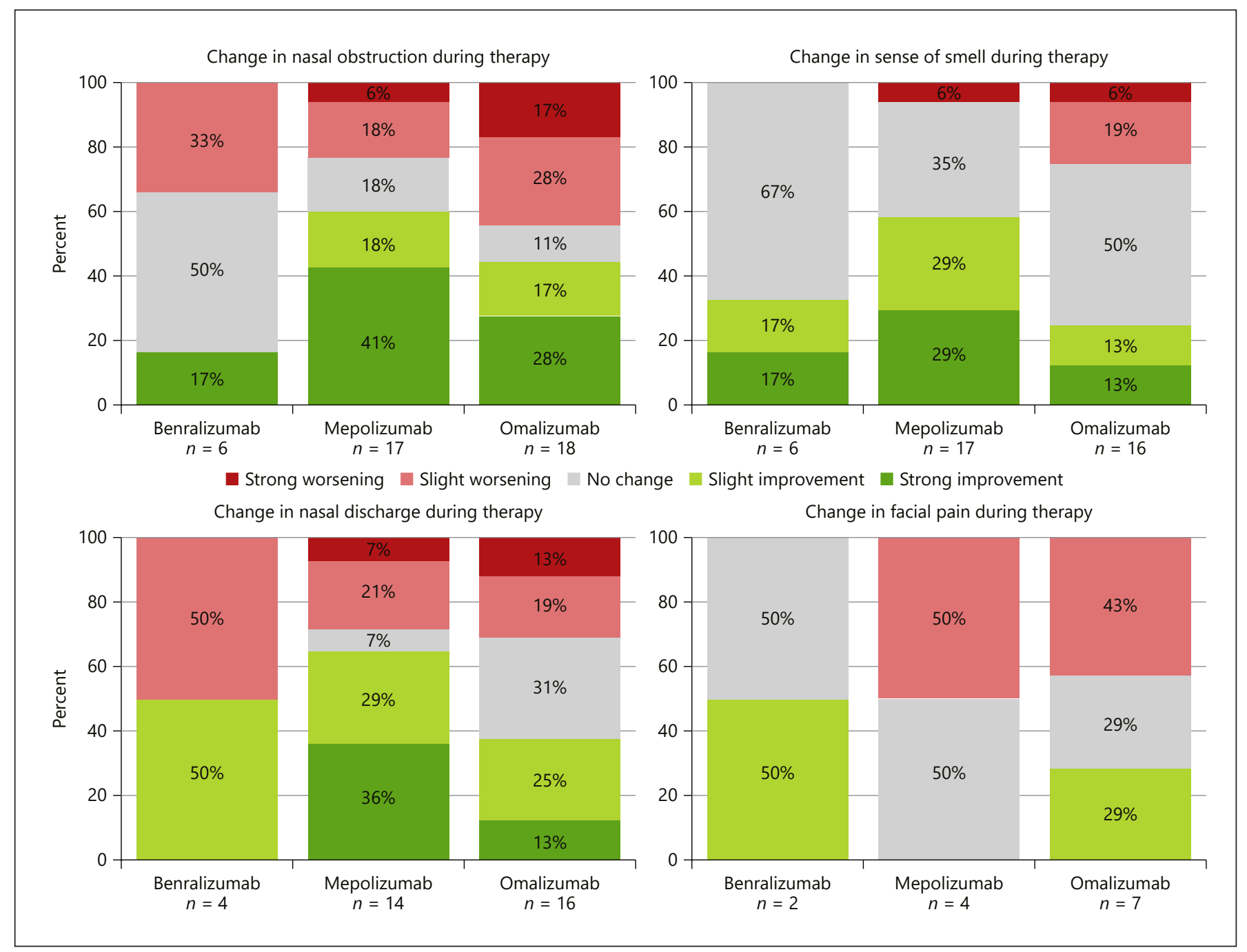

Fig. 4. Change in clinical symptoms during therapy with benralizumab, mepolizumab, or omalizumab.

In our mepolizumab group, nasal congestion and sense of smell improved in 58.8\%, and rhinorrhea in $64.3 \%$. These results are in-line with previous studies [3, 6], which also reported an improvement in these symptoms. A newer study [10], similarly using a lower dosage and subcutaneous route of administration, could not show any improvement in sense of smell, yet, we saw an enhancement. However, we did not directly test the sense of smell with a psychophysical test. Therefore, a bias of better smell perception in patients with better nasal patency could be present, as described by Landis et al. [13].

Of the omalizumab patients, $37.5 \%$ showed a reduction in the polyp score. However, since the polyp score increased on average, it remains difficult to assess treatment success in terms of polyp size alone. The influence of omalizumab on the total polyp score was also controversially discussed in earlier studies. Gevaert et al. [4] and Vennera et al. [7] showed a significant reduction in the total polyp score compared to placebo or baseline, respectively. On the contrary, 2 studies of Pinto et al. [9] and Tiotiu et al. [8] could not show a significant reduction in endoscopic polyp scores. This highlights the necessity to choose the right treatment for the right patient, to ensure the most successful outcome, regardless, due to the lack of predictive markers and clear endotyping this remains a major challenge. In terms of improving clinical symptoms with omalizumab therapy, our results are not very clear either. Compared to previous studies, our results rest between two studies, one which reported a significant improvement in loss of sense of smell, rhinor- 
rhea, and nasal obstruction $[4,8]$, and another that showed no significant difference in improvement of the 20-item Sino-Nasal Outcome Test score, and olfactory function (measured by University of Pennsylvania Smell Identification Test) [9].

Additionally, in patients treated with systemic steroids (as constant daily therapy or as rescue therapy), combined with omalizumab and mepolizumab, treatments seemed to be less successful compared to patients without need of systemic steroids. It can even be said that all our mepolizumab patients without systemic steroids had successful or partially successful treatment. We can interpret this finding that patients in need of systemic steroids have a higher burden of disease and are usually more difficult to treat or have, so far, not received the right treatment [2]. A reduction in frequency of systemic steroids was not analyzed in our study, but Pinto et al. [9] reported a reduced use of systemic rescue steroids in omalizumab patients compared with placebo, although the small number of patients precluded definitive conclusions.

Head et al. demonstrated that some patients showed a good response to short-term treatment with systemic steroids and a tendency for recurrence after stopping [14]. With this in mind, combined with the intention to stabilize the success of a short-term treatment with systemic steroids, in selected cases we started the treatment with $\mathrm{mAB}$ directly after, or even in combination with a shortterm steroid treatment as an induction procedure.

In the mepolizumab group, there was a trend for a higher rate of successful, or partially successful treatments at the beginning of therapy compared to patients without, but the difference of $20.8 \%$ between the two groups was not significant. In the omalizumab group, however, the outcome was the same in both subgroups. Nevertheless, the use of systemic steroids as an induction therapy not only improved the treatment outcome but lead to a more rapid resolution of symptoms and therefore improved the quality of life. Future studies could focus on the effect of induction therapy before the start of $\mathrm{mAB}$ treatments.

Although the course of ECP value was not available in many cases, it seems like a reduction during therapy with mepolizumab correlated with a more successful treatment outcome compared to cases with an ECP value increase during treatment. This is in-line with a previous study, which indicated simultaneously a significant reduction in the polyp score and ECP value under treatment with mepolizumab compared to placebo [3]. Unfortunately, ECP does not seem to predict the therapeutic outcome with $\mathrm{mAB}$ treatment.
Due to the retrospective nature and the "real-life" setting of this study, we have to mention some limitations. First, in Switzerland, mABs are not approved for the treatment of CRSwNP. With the exception of 1 patient, all our patients were treated with a $\mathrm{mAB}$ when diagnosed with a simultaneous asthma. Therefore, no statement can be made on treatment success in CRSwNP-patients without asthma. Second, the different group sizes reflect the time of market approval of mepolizumab and omalizumab in Switzerland. Therefore, we had to accept a smaller benralizumab group with only 6 cases in this group. Although other studies showed a significant decrease in the SNOT-22 score and endoscopic polyp score, the small group size in our study made it nearly impossible to see a real trend of treatment success $[15,16]$. We were also unable to include dupilumab, which also appears to be a very promising biological compound in the treatment of CRSwNP [17].

\section{Conclusion}

Specific $\mathrm{mAB}$ therapies seem to be another attractive option for treating CRSwNP in difficult-to-treat patients. However, little is known about benralizumab as a possibility. Due to missing predictive biomarkers, it still remains unknown how the right $\mathrm{mAB}$ for an individual patient can be selected. Decreased eosinophil activity as measured by ECP seems to have an important effect in responders, while sensitizations to allergens or blood eosinophils in our collective do not correlate with the observed outcome. Furthermore, it is unknown, how long a therapy with $\mathrm{mABs}$ should be carried out if the treatment is successful.

\section{Statement of Ethics}

This study was approved by the Ethical Committee of the Canton Zurich (KEK-2019-00495). Every patient signed a general informed consent, or in case of additional diagnostics due to the study, a study specific consent was obtained.

\section{Conflict of Interest Statement}

M.B.S. acted as a consultant in a study on Dupilumab and received consulting fees by Biomedical Systems/Sanofi. P.S.G. has received speaker fees from AstraZeneca, GSK, and SanofiGenzyme. No other author has reported any competing interests. 


\section{Funding Sources}

This research did not receive any specific grant from funding agencies in the public, commercial, or not-for-profit sectors.

\section{Author Contributions}

E.C.M. drafted the manuscript and performed the data analysis and interpretation with the help of M.B.S., U.C.S., and P.S.G., who reviewed the manuscript. All authors read and approved the final manuscript.

\section{References}

1 Hastan D, Fokkens WJ, Bachert C, Newson $\mathrm{RB}$, Bislimovska J, Bockelbrink A, et al. Chronic rhinosinusitis in Europe-an underestimated disease. A GA(2)LEN study. Allergy. 2011;66(9):1216-23.

2 Fokkens WJ, Lund VJ, Hopkins C, Hellings PW, Kern R, Reitsma S, et al. European position paper on rhinosinusitis and nasal polyps 2020. Rhinology. 2020;58(Suppl S29):1-464.

3 Gevaert P, Van Bruaene N, Cattaert T, Van Steen K, Van Zele T, Acke F, et al. Mepolizum$\mathrm{ab}$, a humanized anti-IL-5 mAb, as a treatment option for severe nasal polyposis. J Allergy Clin Immunol. 2011;128(5):989-95.e1-8.

4 Gevaert P, Calus L, Van Zele T, Blomme K, De Ruyck N, Bauters W, et al. Omalizumab is effective in allergic and nonallergic patients with nasal polyps and asthma. J Allergy Clin Immunol. 2013;131(1):110-6.e1.

5 Bachert C, Mannent L, Naclerio RM, Mullol J, Ferguson BJ, Gevaert P, et al. Effect of subcutaneous dupilumab on nasal polyp burden in patients with chronic sinusitis and nasal polyposis: a randomized clinical trial. JAMA. 2016;315(5):469-79.

6 Bachert C, Sousa AR, Lund VJ, Scadding GK, Gevaert P, Nasser S, et al. Reduced need for surgery in severe nasal polyposis with mepolizumab: randomized trial. J Allergy Clin Immunol. 2017;140(4):1024-31.e14.
7 Vennera MC, Picado C, Mullol J, Alobid I, Bernal-Sprekelsen M. Efficacy of omalizumab in the treatment of nasal polyps. Thorax. 2011;66(9):824-5.

8 Tiotiu A, Oster JP, Roux PR, Nguyen Thi PL, Peiffer G, Bonniaud P, et al. Effectiveness of omalizumab in severe allergic asthma and nasal polyposis: a real-life study. J Investig Allergol Clin Immunol. 2020;30(1):49-57.

9 Pinto JM, Mehta N, DiTineo M, Wang J, Baroody FM, Naclerio RM. A randomized, double-blind, placebo-controlled trial of anti-IgE for chronic rhinosinusitis. Rhinology. 2010; 48(3):318-24.

10 Chan R, RuiWen Kuo C, Lipworth B. Disconnect between effects of mepolizumab on severe eosinophilic asthma and chronic rhinosinusitis with nasal polyps. J Allergy Clin Immunol Pract. 2020;8(5):1714-6.

11 Shomali W, Gotlib J. World Health Organization-defined eosinophilic disorders: 2019 update on diagnosis, risk stratification, and management. Am J Hematol. 2019;94(10): 1149-67.

12 Brozek JL, Bousquet J, Agache I, Agarwal A, Bachert C, Bosnic-Anticevich S, et al. Allergic rhinitis and its impact on asthma (ARIA) guidelines-2016 revision. J Allergy Clin Immunol. 2017;140(4):950-8.
13 Landis BN, Hummel T, Hugentobler $M$, Giger R, Lacroix JS. Ratings of overall olfactory function. Chem Senses. 2003;28(8):6914.

14 Head K, Chong LY, Hopkins C, Philpott C, Burton MJ, Schilder AG. Short-course oral steroids alone for chronic rhinosinusitis. Cochrane Database Syst Rev. 2016; 4: CD011991.

15 Chitguppi C, Patel P, Gandler A, Murphy K, Khoury Md T, Monostra P, et al. Effect of benralizumab in patients with severe eosinophilic asthma and chronic rhinosinusitis with nasal polyps: a case series. Am J Rhinol Allergy. 2020:1945892420978351.

16 Lombardo N, Pelaia C, Ciriolo M, Della Corte M, Piazzetta G, Lobello N, et al. Real-life effects of benralizumab on allergic chronic rhinosinusitis and nasal polyposis associated with severe asthma. Int $\mathrm{J}$ Immunopathol Pharmacol. $2020 \quad$ Jan-Dec; 34: 2058738420950851.

17 Bachert C, Han JK, Desrosiers M, Hellings PW, Amin N, Lee SE, et al. Efficacy and safety of dupilumab in patients with severe chronic rhinosinusitis with nasal polyps (LIBERTY NP SINUS-24 and LIBERTY NP SINUS-52): results from two multicentre, randomised, double-blind, placebo-controlled, parallelgroup phase 3 trials. Lancet. 2019;394(10209): 1638-50. 